\title{
ON A UNIQUENESS PROPERTY OF SECOND CONVOLUTIONS
}

\author{
N. BLANK
}

\section{Introduction and Main Result}

Let $M_{\infty}$ denote the space of all finite nontrivial complex Borel measures on the real line whose variation has a fast decay at $-\infty$ :

$$
\int_{-\infty}^{0} e^{r|t|} d|\mu(t)|<\infty, \quad \text { for every } \quad r>0 .
$$

It follows from (1) that the Fourier-Stieltjes transform of every measure $\mu \epsilon$ $M_{\infty}$

$$
\hat{\mu}(z):=\int_{-\infty}^{\infty} e^{i z t} d \mu(t),
$$

converges uniformly on compact subsets of the upper half-plane $C_{+}:=\{z \in$ $\mathrm{C}: \operatorname{Im} z>0\}$ to a function analytic in $C_{+}$. Let $l(\mu):=\inf \operatorname{supp} \mu$ denote the left boundary of the support of $\mu$, and $\mu^{n *}$ the $n$th convolution power of $\mu$.

The following uniqueness property of $n$th convolutions of measures from $M_{\infty}$ was discovered in connection with some probabilistic results (see for example [1], [7], [8], [9], [10] and the literature therein): Let $n \geq 3$ be an integer, and let $\mu \in M_{\infty}$ be such that $l(\mu)=-\infty$. Then every half-line $(-\infty, a), a \in \mathrm{R}$, is a uniqueness set for the $n$th convolution $\mu^{n *}$, in the sense that the implication holds: Suppose $v \in M_{\infty}$ and

(2) there exists $a \in \mathrm{R}$ such that $\left.\mu^{n *}\right|_{(-\infty, a)}=\left.v^{n *}\right|_{(-\infty, a)}$. Then $\mu^{n *}=v^{n *}$.

It is also known that property (2) does not hold for $n=2$. An easy way to check this is to take two measures $\xi_{1}, \xi_{2} \in M_{\infty}$ such that $l\left(\xi_{1}+\xi_{2}\right)=-\infty$ and $\xi_{1} * \xi_{2}=0$ on some half-line $(-\infty, a)$. Then the measures $\mu=\xi_{1}+\xi_{2}$ and $v=\xi_{1}-\xi_{2}$ belong to $M_{\infty}, l(\mu)=-\infty$ and we have

$$
\left.\left(\mu^{2 *}-v^{2 *}\right)\right|_{(-\infty, a)}=\left.4 \xi_{1} * \xi_{2}\right|_{(-\infty, a)}=0 .
$$

Received August 15, 2007. 
For example, let $\xi_{j} \in M_{\infty}$ be the measures with Fourier-Stieltjes transforms

$$
\hat{\xi}_{j}(z)=e^{(-1)^{j} e^{-i z}}, \quad j=1,2 .
$$

From $\hat{\xi}_{1} \hat{\xi}_{2}=1$, we see that $\xi_{1} * \xi_{2}$ is the unit measure concentrated at the origin, so that $\left(\xi_{1}+\xi_{2}\right)^{2 *}-\left(\xi_{1}-\xi_{2}\right)^{2 *}=4 \xi_{1} * \xi_{2}=0$ on $(-\infty, 0)$.

It turns out that there cannot be more than two different second convolutions which agree on a half-line. The aim of this note is to prove the following

Theorem 1. Assume a measure $\mu \in M_{\infty}$ satisfies $l(\mu)=-\infty$. Suppose there exists $a \in \mathrm{R}$ and measures $v, \phi \in M_{\infty}$ such that

$$
\left.\mu^{2 *}\right|_{(-\infty, a)}=\left.v^{2 *}\right|_{(-\infty, a)}=\left.\phi^{2 *}\right|_{(-\infty, a)},
$$

and $v^{2 *} \neq \phi^{2 *}$. Then either $v^{2 *}=\mu^{2 *}$ or $\phi^{2 *}=\mu^{2 *}$.

An immediate corollary is the following uniqueness property of the second convolutions:

CoROllary 2. For every $\mu \in M_{\infty}, l(\mu)=-\infty$, there is a real number $a_{0}=a_{0}(\mu)$ such that $\mu^{2 *}$ is uniquely determined by its values on $(-\infty, a), a>$ $a_{0}$, i.e. if $v \in M_{\infty}$ and there exists $a>a_{0}$ such that $\left.\mu^{2 *}\right|_{(-\infty, a)}=\left.v^{2 *}\right|_{(-\infty, a)}$, then $\mu^{2 *}=v^{2 *}$.

We also mention a uniqueness result for squares of analytic functions:

COROLlary 3. Assume functions $f, g$ and $h$ are analytic in the punctured unit disk $0<|z|<1$, and that $f$ has an essential singularity at the origin. Suppose that both functions $f^{2}-g^{2}$ and $f^{2}-h^{2}$ have a pole or a removable singularity at the origin and $g^{2} \neq h^{2}$. Then either $g^{2}=f^{2}$ or $h^{2}=f^{2}$.

This is just a particular case of Theorem 1 for measures concentrated on the set of integers, and follows from it by the change of variable $z=\exp (-i t)$.

\section{Remarks}

1. Observe that condition (1) is crucial for the uniqueness property (2): The property (2) does not in general hold for measures whose Fourier-Stieltjes transform is not analytic in $C_{+}$, see [7], [8] and [1]. A comprehensive survey of results on this and similar uniqueness properties can be found in [9].

2. As it was observed in [7], the uniqueness property of $n$th convolutions (2) is closely connected with the Titchmarsh convolution theorem and its extensions. The classical Titchmarsh convolution theorem states that if $\xi_{1}$ and $\xi_{2}$ are finite Borel measures satisfying $l\left(\xi_{j}\right)>-\infty, j=1,2$, then $l\left(\xi_{1} * \xi_{2}\right)=l\left(\xi_{1}\right)+l\left(\xi_{2}\right)$. This is not true for measures with unbounded support, 
for there exist measures $\xi_{j}, j=1,2, l\left(\xi_{1}\right)=-\infty$, such that $l\left(\xi_{1} * \xi_{2}\right)>-\infty$. Such measures can be taken from $M_{\infty}$, see example (3). However, it was shown in [8] that the conclusion of Titchmarsh convolution theorem holds true whenever the variation of measures satisfies a condition at $-\infty$ more restrictive than (1):

$$
\int_{-\infty}^{0} e^{r|t| \log |t|} d|\mu(t)|<\infty, \quad \text { for every } \quad r>0 .
$$

Second convolutions of such measures enjoy the uniqueness property above ([7], [8]). Moreover, examples similar to (3) show that restriction (5) cannot be weakened. Analogous results for unbounded measures were established in [2].

Observe that extensions of the Titchmarsh convolution theorem have also applications in the theory of invariant subspaces, see [2], [3] and [4].

3 . The Titchmarsh convolution theorem has been extended to linearly dependent measures: the equality

$$
l\left(\xi_{1} * \cdots * \xi_{n}\right)=\sum_{j=1}^{n} l\left(\xi_{j}\right)
$$

holds for linearly dependent measures $\xi_{j} \in M_{\infty}, j=1, \ldots, n, n \geq 3$, in "general position", for the precise statement see [5]. Our proof of Theorem 1 below is a fairly easy consequence of this result.

\section{Proof of Theorem 1}

The following lemma is a particular case of Theorem 4 in [5]:

Lemma 4. (i) Suppose measures $\xi_{1}, \xi_{2}, \xi_{3} \in M_{\infty}$ are linearly independent over C. Then

(6) $l\left(\xi_{1} * \xi_{2} * \xi_{3} *\left(\xi_{1}+\xi_{2}+\xi_{3}\right)\right)=l\left(\xi_{1}\right)+l\left(\xi_{2}\right)+l\left(\xi_{3}\right)+l\left(\xi_{1}+\xi_{2}+\xi_{3}\right)$.

(ii) Suppose measures $\xi_{1}, \xi_{2} \in M_{\infty}$ are linearly independent over $\mathrm{C}$ and $\left|a_{1}\right|+\left|a_{2}\right| \neq 0$. Then

$$
\begin{aligned}
l\left(\xi_{1} * \xi_{2} *\left(\xi_{1}+\xi_{2}\right) *\left(a_{1} \xi_{1}+a_{2} \xi_{2}\right)\right) & \\
& =l\left(\xi_{1}\right)+l\left(\xi_{2}\right)+l\left(\xi_{1}+\xi_{2}\right)+l\left(a_{1} \xi_{1}+a_{2} \xi_{2}\right) .
\end{aligned}
$$

For the convenience of the reader, we recall shortly the main ideas of the proof in [5]. To prove, say (6), by the Titchmarsh convolution theorem, it 
suffices to verify the implication

$$
l\left(\xi_{1} * \xi_{2} * \xi_{3} *\left(\xi_{1}+\xi_{2}+\xi_{3}\right)\right)>-\infty \Rightarrow l\left(\xi_{j}\right)>-\infty, \quad j=1,2,3 .
$$

We may assume that $\xi_{1} * \xi_{2} * \xi_{3} *\left(\xi_{1}+\xi_{2}+\xi_{3}\right)=0$ on $(-\infty, 0)$, so that the product of the Fourier-Stieltjes transforms $\hat{\xi}_{1} \hat{\xi}_{2} \hat{\xi}_{3}\left(\hat{\xi}_{1}+\hat{\xi}_{2}+\hat{\xi}_{3}\right)$ belongs to the Hardy space $H^{\infty}\left(\mathrm{C}_{+}\right)$. Hence, the zero set of the product, and so the zero set of each factor satisfies the Blaschke condition. Now one can use the following argument: If functions $f_{j}, j=1, \ldots, n, n \geq 2$, are analytic in the unit disk, linearly independent and such that the zeros of each $f_{j}$ and the sum $f_{1}+\cdots+f_{n}$ satisfy the Blaschke condition in the disk, then each $f_{j}$ must have "slow" growth in the disk. A sharp statement follows from H. Cartan's second main theorem for analytic curves, see Theorem $D$ in [5]. This argument proves that the growth of each $\hat{\xi}_{j}$ in $C_{+}$must satisfy a certain restriction. Next, we have additional information that each function $\hat{\xi}_{j}$ is bounded in every horizontal strip in $\mathrm{C}_{+}$. This allows one to improve the previous estimate to show that numbers $b_{j}$ exist such that $\hat{\xi}_{j}(z) \exp \left(i b_{j} z\right) \in H^{\infty}\left(\mathrm{C}_{+}\right), j=1,2,3$. This means that $l\left(\xi_{j}\right) \geq-b_{j}>-\infty, j=1,2,3$.

We shall also need a simple lemma:

Lemma 5. Suppose $\mu \in M_{\infty}$ is such that $l\left(\mu^{2 *}\right)>-\infty$. Then $l(\mu)>-\infty$.

Indeed, we may assume that $\mu^{2 *}=0$ on $(-\infty, 0)$, so that $(\hat{\mu})^{2} \in H^{\infty}\left(\mathrm{C}_{+}\right)$. Since $\hat{\mu}$ is analytic in $\mathrm{C}_{+}$, we obtain $\hat{\mu} \in H^{\infty}\left(\mathrm{C}_{+}\right)$. Consider now convolutions $\mu * p_{n}$, where $p_{n}$ is any sequence of smooth functions concentrated on $[0, \infty]$ which converges weakly to the delta-function concentrated at the origin. We have $\hat{p}_{n} \hat{\mu} \in\left(H^{\infty} \cap H^{1}\right)\left(\mathrm{C}_{+}\right)$. A standard argument involving inverse Fourier transform along the line $\operatorname{Im} z=y$ as $y \rightarrow \infty$, proves that $l\left(\mu * p_{n}\right) \geq 0$. Taking the limit as $n \rightarrow \infty$, we conclude that $l(\mu) \geq 0$.

Proof of Theorem 1. Suppose measures $\mu, v, \phi \in M_{\infty}, l(\mu)=-\infty$, satisfy (4) for some $a \in \mathrm{R}$, and $v^{2 *} \neq \phi^{2 *}$. Set $\xi_{1}:=(\mu+v) / 2, \xi_{2}:=$ $(\mu-v) / 2$, and $\eta_{1}:=(\mu+\phi) / 2, \eta_{2}:=(\mu-\phi) / 2$. To prove the theorem, it suffices to show that one of the measures $\xi_{j}, \eta_{j}, j=1,2$, is trivial.

Let us assume that it is not so, and show that this leads to a contradiction. Since

$$
\begin{gathered}
\left.\left(\mu^{2 *}-v^{2 *}\right)\right|_{(-\infty, a)}=\left.4 \xi_{1} * \xi_{2}\right|_{(-\infty, a)}=0, \\
\left.\left(\mu^{2 *}-\phi^{2 *}\right)\right|_{(-\infty, a)}=\left.4 \eta_{1} * \eta_{2}\right|_{(-\infty, a)}=0,
\end{gathered}
$$

we have

$$
l\left(\xi_{1} * \xi_{2}\right)>-\infty, l\left(\eta_{1} * \eta_{2}\right)>-\infty .
$$


Let us show that (7) implies $l(\mu)>-\infty$, which contradicts the assumption $l(\mu)=-\infty$.

We shall consider several cases. First, assume that $\xi_{1}$ and $\xi_{2}$ are linearly dependent. Then $\mu=\xi_{1}+\xi_{2}=(1+b) \xi_{2}$, for some $b \in \mathrm{C}, b \neq 0$, and so

$$
\mu^{2 *}=(1+b)^{2} \xi_{2}^{2 *}=\frac{(1+b)^{2}}{b} \xi_{1} * \xi_{2} .
$$

By (7), this gives $l\left(\mu^{2 *}\right)>-\infty$. Lemma 5 yields $l(\mu)>-\infty$.

Assume now that $\xi_{1}$ and $\xi_{2}$ are linearly independent. From $\mu=\xi_{1}+\xi_{2}=$ $\eta_{1}+\eta_{2}$ we have $\eta_{2}=\xi_{1}+\xi_{2}-\eta_{1}$. Now (7) gives

$$
-\infty<l\left(\xi_{1} * \xi_{2} * \eta_{1} * \eta_{2}\right)=l\left(\xi_{1} * \xi_{2} * \eta_{1} *\left(\xi_{1}+\xi_{2}-\eta_{1}\right)\right) .
$$

If $\xi_{1}, \xi_{2}$ and $\eta_{1}$ are linearly independent, then by part (i) of Lemma 4, we obtain $l\left(\xi_{j}\right)>-\infty, j=1,2$, and so $l(\mu)>-\infty$. If $\xi_{1}, \xi_{2}$ and $\eta_{1}$ are linearly dependent, we have $\eta_{1}=c_{1} \xi_{1}+c_{2} \xi_{2}$, for some $c_{1}, c_{2} \in \mathrm{C}$. Hence,

$$
-\infty<l\left(\xi_{1} * \xi_{2} * \eta_{1} * \eta_{2}\right)=l\left(\xi_{1} * \xi_{2} *\left(c_{1} \xi_{1}+c_{2} \xi_{2}\right)\right) *\left(\left(1-c_{1}\right) \xi_{1}+\left(1-c_{2}\right) \xi_{2}\right) .
$$

If either $c_{j} \neq 0, j=1,2$, or $1-c_{j} \neq 0, j=1,2$, then part (ii) of Lemma 4 implies $l\left(\xi_{j}\right)>-\infty$, and so $l(\mu)>-\infty$. Otherwise, we may assume that $c_{1}=0$ and $1-c_{2}=0$. This gives

$$
-\infty<l\left(\xi_{1} * \xi_{2} * \eta_{1} * \eta_{2}\right)=l\left(\xi_{1}^{2 *} * \xi_{2}^{2 *}\right) .
$$

From (7) and Lemma 5 we conclude that $l\left(\xi_{j}\right)>-\infty, j=1,2$, which shows that $l(\mu)>-\infty$.

\section{REFERENCES}

1. Blank, N. M., Distributions whose convolutions coincide on the half axis. (Russian) Teor. Funktsiu Funktsional. Anal. i Prilozhen. No. 41 (1984), 17-25.

2. Borichev, A. A., The generalized Fourier transform, the Titchmarsh theorem and almost analytic functions. (Russian) Algebra i Analiz 1 (1989), no. 4, 17-53; translation in Leningrad Math. J. 1 (1990), 825-857.

3. Borichev, A. A., Errata: "The generalized Fourier transform, the Titchmarsh theorem and almost analytic functions". Algebra i Analiz 2 (1990), no. 5, 236-237.

4. Domar, Y., Extension of the Titchmarsh convolution theorem with applications in the theory of invariant subspaces, Proc. London. Math. Soc. (3) 46 (1983), 288-300.

5. Gergün, S., Ostrovskii, I. V., Ulanovskii, A., On the Titchmarsh convolution theorem. Ark. Mat. 40 (2002), 55-71.

6. Hayman, W. K., Subharmonic Functions. Vol. 2, London Math. Soc. Monographs 20, Academic Press, London 1989. 
7. Ostrovskii, I. V., Support of a convolution of finite measures, and measures determined uniquely by the restriction to a half-line. (Russian) Dokl. Akad. Nauk Ukrain. SSR Ser. A 1984, no. 3, 8-12.

8. Ostrovskii, I. V., Generalization of the Titchmarsh convolution theorem and the complexvalued measures uniquely determined by their restrictions to a half-line. pp. 256-283 in Stability Problems for Stochastic Models (Uzhgorod, 1984), Lecture Notes in Math. 1155, Springer, Berlin 1985.

9. Ostrovskii, I. V., Ulanovskii, A., Classes of complex-valued Borel measures that can be uniquely determined by restrictions. (Russian) Zap. Nauchn. Sem. Leningrad. Otdel. Mat. Inst. Steklov. (LOMI) 170 (1989), Issled. Linein. Oper. Teorii Funktsii. 17, 233-253, 325; translation in J. Soviet Math. 63 (1993), 246-257.

10. Ramachandran, B., On the results of Ibragimov, Titov and Blank on distribution functions on the real line-their convolution powers coinciding on a half-line, J. Indian Statist. Assoc. 36 (1998), 75-81.

STAVANGER UNIVERSITY

4036 STAVANGER

NORWAY

E-mail: Natalia.Blank@uis.no 